\title{
Sandpiles and absorbing-state phase transitions: recent results and open problems
}

\author{
Miguel A. Muñoz ${ }^{1}$, Ronald Dickman ${ }^{2}$, Romualdo Pastor-Satorras \\ ${ }^{3}$, Alessandro Vespignani ${ }^{4}$, and Stefano Zapperi ${ }^{5}$ \\ ${ }^{1}$ Institute Carlos I for Theoretical and Computational Physics \\ and Depto. de E. y Física de la Materia, Universidad de Granada, 18071 Granada, Spain. \\ ${ }^{2}$ Departamento de Física, ICEx, Universidade Federal de Minas Gerais, Caixa Postal 702, \\ 30161-970 Belo Horizonte, MG, Brazil \\ ${ }^{3}$ Dept. de Física i E. Nuclear, Universitat Politècnica de Catalunya, Campus Nord, Módul B4, \\ 08034 Barcelona, Spain \\ ${ }^{4}$ The Abdus Salam Int. Centre for Theoretical Physics P.O. Box 586, 34100 Trieste, Italy \\ ${ }^{5}$ INFM, Dipartimento di Fisica, E. Fermi, Universitá de Roma "La Sapienza", P.le A. Moro 2, \\ 00185 Roma, Italy
}

\begin{abstract}
We review some recent results on the relations between sandpiles and a class of absorbing state phase transitions. We use the concept of fixed energy sandpiles (FES), in which external driving and dissipation are absent. FES are shown to exhibit an absorbing state transition with critical properties coinciding with those of the corresponding sandpile model. We propose a set of Langevin equations capturing the relevant features of this transition. These equations characterize the universality class of systems with an infinite number of absorbing states and a static conserved field coupled to the order parameter. Different models in this class are identified, and strong evidence is presented showing that the Manna sandpile, as well as some other stochastic sandpiles, belong in this universality class. Finally some open problems and questions are discussed.
\end{abstract}

\section{INTRODUCTION}

Slowly driven by the input of new ideas and concepts, avalanches of papers have in recent years seen the light in the field of self-organized criticality (SOC) [1-8]. This activity was certainly justified by the huge conceptual and practical implications of the self-organization mechanisms. From a theoretical point of view 
two main questions were soon posed: (i) what are the main ingredients able to drive "spontaneously" a system to its critical state without need of fine-tuning any control parameter?, and (ii) what are the links between self-organized critical states and standard critical ones appearing in equilibrium and non-equilibrium systems?. In order to answer the first of the two aforementioned issues, several paths to SOC have been identified (a recent review of them, as well as a summary on experiments on SOC can be found in [8]). Certainly the most celebrated ones among them are: the presence of two infinitely-separated time scales [6], and extremal dynamics [9], though these are certainly not the only ones $[4,8]$.

For example, in sandpiles [1-3], the archetype of SOC systems, the two infinitely separated time scales correspond to a slow scale at which grains of sand are added to the system, and a fast one for the relaxation of unstable piles which leads to a rapid redistribution of sand, and eventually to boundary dissipation. It has been shown that it is only in the double limit of driving rate, $h$, and dissipation rate, $\epsilon$ going to $0^{+}$with $h / \epsilon \rightarrow 0$, that scale invariant behavior is generated up to the system-size cut-off $[6,10]$. Under the effect of the external drive, the system jumps among stable (absorbing) configurations via avalanche-like rearrangements. Some of the sandpiles we will refer to are: the original Bak-Tang-Wiesenfeld (BTW) sandpile [1], the Manna model [2], the Zhang model [3], the Oslo ricepile model [11], as well as some variations of them.

Despite of some very remarkable rigorous results $[4,12]$ and some renormalization group analyses [13], the question of whether the critical properties of sandpiles can be related to more standard critical phase transitions, or more specifically, whether the associated critical (avalanche) exponents $[1,2,5]$ can be ascribed to any known universality class, remains open.

A priori, these are not straightforward questions to answer; in particular, the presence of two time scales makes it difficult to apply standard techniques for the study of scale invariant systems to sandpiles. Another intrinsic difficulty lies in the fact, that exponents controlling the deviation from criticality cannot be measured in slowly driven sandpiles, posed by definition at their critical point.

\section{FIXED ENERGY SANDPILES AND ABSORBING STATES}

In order to circumvent these difficulties we have studied the the so-called fixed energy sandpiles (FES) [14-16]. These are cellular automata sharing the same microscopic dynamics as their corresponding standard sandpile counterparts but without external driving or dissipation. For each standard sandpile model we can define its FES version by keeping constant its total energy (number of sand grains). In this way, the initial energy density $\zeta$ becomes a control parameter. FES are found to be critical only for a particular value $\zeta=\zeta_{c}$ (which as we will show turns out to be identical to the stationary energy density of its driven-dissipative counterpart 
[16]). For energy densities below $\zeta_{c}$ the FES-sandpile falls into an absorbing state [17], while for values above that threshold activity is indefinitely sustained, i.e. the FES is in an active phase. Consequently, the phase transition in FES can be enviewed as an absorbing state phase transition [17,19] (with an infinite number of absorbing states). Observe that, switching on dissipation and driving, in the presence of activity $\zeta>\zeta_{c}$ and $d \zeta / d t<0$. In the absence of activity there is addition, but no loss of activity, so $\zeta<\zeta_{c}$ implies $d \zeta / d t>0$ [18]. Evidently, the only possible stationary value for the density in the sandpile is $\zeta_{c}$ ! Not only $\zeta_{c}$ is common to a critical FES and its slowly-driven counterpart: also higher moments and correlation functions coincide in the large-size limit (as expected, given that the bulk dynamics is identical in both cases). We have performed extensive numerical analyses of the critical behavior of FES aimed at verifying the previous arguments and at determining a complete set of exponents. We refer the reader to [16] where the main ideas, simulations, analysis techniques, and results are presented (some of the obtained critical exponents are included in table 1).

Let us remark that other attempts have been made to connect sandpiles with different types of phase transitions, as for instance: pinning-depinning transitions in disordered media [20], the voter model [21], and branching processes [22]. It is beyond the scope of this paper to review or discuss them.

Generically systems exhibiting a transition into an absorbing state belong in the universality class of directed percolation (DP), the field theoretical (or Langevin equation) representation of which is the celebrated Reggeon field theory (RFT) $[23,17]$. This universality class has proven very robust with respect to the variation of microscopic details, number of components, and number of absorbing states $[24,25,17]$. Only in the presence of some extra conservation law or additional symmetry (as, for example, a $Z_{2}$-symmetry between two equivalent absorbing states, which in some cases tantamount to parity conservation [26]), have the critical exponents been found to differ from those of DP [26].

Following rather general principles of relevance and symmetry, a set of Langevin equations aimed at capturing the critical features of FES was proposed in [16]. Let us emphasize that this type of phenomenological approach has proven extremely useful, and provided sound results when applied to other systems with many absorbing states (see [25]). This set of equations includes one for the evolution of a coarse-grained activity field, $\phi(\mathbf{x}, t)$, and one for the energy (or background) field, $E(\mathbf{x}, t)[16]$. The activity field represents the local density of unstable sites, while the energy field stands for the total number of grains regardless of the local activity state. The proposed set of equations is:

$$
\begin{aligned}
\partial_{t} \phi(\mathbf{x}, t) & =\mu \phi(\mathbf{x}, t)-b \phi^{2}(\mathbf{x}, t)+D \nabla^{2} \phi(\mathbf{x}, t)+w E(\mathbf{x}, t) \phi(\mathbf{x}, t)+\sigma \sqrt{\phi} \eta(\mathbf{x}, t) \\
\partial_{t} E(\mathbf{x}, t) & =\lambda \nabla^{2} \phi(\mathbf{x}, t)
\end{aligned}
$$

where $\mu, b, D, w, \sigma$, and $\lambda$ are constants, and $\eta$ is a Gaussian white noise. In a nutshell, these equations express the fact that the transition for the activity is controlled by the same type of terms appearing in the RFT, i.e. the most rele- 
vant terms in standard absorbing-state phase transitions $[17,23]$, plus an additional coupling between the activity field and a static conserved energy field. This extra term stems from the fact that creation of activity is locally fostered by the presence of a high density of the background field, and this background field (energy) is a conserved one. The extra conservation law is therefore the new relevant ingredient with respect to RFT. Some other terms, consistent with the symmetries and conservation laws, could have been included in Eq.(1) but they all turn out to be irrelevant from a power counting analysis [16]. Let us also remark that the second equation in Eq.(1), being linear, can be integrated out, leading to a non-Markovian contribution and a quenched disordered linear term in the activity equation (a detailed analysis and discussion of these terms can be found in [16]). In what follows, we avoid performing such an integration to make explicit the presence of a conservation law, and stress that this extra contribution is relevant at the RFT fixed point [16].

Which sandpiles is this theory intended to describe? Although in principle we would like to describe the original BTW sandpile this seems to be an unrealizable task, the reasons for this being manifold. The main reason, is that the BTW model being deterministic, has many hidden conservation laws and toppling invariants [4] which are almost impossible to implement in a coarse grained Langevin equation (for which a detailed book-keeping of symmetries and conservation laws is essential). Owing to the existence of these conservation laws, the FES version of the BTW exhibits strong non-ergodicity as shown and discussed in [16]; the stationary state depends strongly upon the initial configuration (and not only on the initial energy). From a different perspective, it has also been recently shown that BTW does not obey simple scaling [7].

Our approach is therefore intended to reproduce the scaling behavior of stochastic sandpiles with no extra conservation, symmetry, or spatial anisotropy [21], as for example: the Manna model [2], ricepiles [11], the stochastic version of the Zhang model $[3,27]$, and other modifications of them.

\section{THE UNIVERSALITY CLASS}

Having proposed a set of Langevin equations for stochastic sandpiles, it would be an extremely desirable next step to derive specific predictions (i.e. values of the critical exponents and scaling functions), obtained after solving perturbatively the renormalization group associated with Eq.(1). Despite considerable efforts, unfortunately, so far we have not been able to complete satisfactorily such a challenging task. Elucidating whether this is just due to technicalities, or has some more profound origin (concerning the theory renormalizability) still needs to be sorted out.

Leaving aside for the moment field theoretical analysis of Eq.(1), we proceed with a slightly more indirect verification of the validity of our theory. Inspired by the previous set of equations Eq.(1) two of us have recently made the following con- 


\begin{tabular}{|c|c|c|c|c|c|c|}
\hline & \multicolumn{6}{|c|}{ Steady state exponents $d=2$} \\
\hline & $\beta$ & $\nu_{\perp}$ & $\beta / \nu_{\perp}$ & $z$ & & $\theta$ \\
\hline CTTP & $0.64(1)$ & $0.82(3)$ & $0.78(3)$ & $1.55(5)$ & & $0.43(1)$ \\
\hline CRD & $0.65(1)$ & $0.83(3)$ & $0.78(2)$ & $1.55(5)$ & & $0.49(1)$ \\
\hline Manna & $0.64(1)$ & $0.82(3)$ & $0.78(2)$ & $1.57(4)$ & & $0.42(1)$ \\
\hline \multirow[t]{3}{*}{ DP } & $0.583(4)$ & $0.733(4)$ & $0.80(1)$ & $1.766(2)$ & & $0.451(1)$ \\
\hline & \multicolumn{6}{|c|}{ Spreading exponents $d=2$} \\
\hline & $\tau_{s}$ & $D$ & $\tau_{t}$ & $z$ & $\eta$ & $\delta$ \\
\hline$\overline{\mathrm{CTTP}}$ & $1.28(1)$ & $2.76(1)$ & $1.49(1)$ & $1.54(2)$ & $0.29(5)$ & $0.50(2)$ \\
\hline CRD & $1.28(1)$ & $2.75(1)$ & $1.50(2)$ & $1.54(2)$ & $0.29(2)$ & $0.50(2)$ \\
\hline Manna & $1.28(1)$ & $2.76(1)$ & $1.48(2)$ & $1.55(1)$ & $0.30(3)$ & $0.48(2)$ \\
\hline \multirow[t]{3}{*}{ DP } & $1.268(1)$ & $2.968(1)$ & $1.450(1)$ & $1.766(2)$ & $0.230(1)$ & $0.451(1)$ \\
\hline & \multicolumn{6}{|c|}{ Steady state exponents $d=3$} \\
\hline & $\beta$ & $\nu_{\perp}$ & $\beta / \nu_{\perp}$ & $z$ & & \\
\hline$\overline{\mathrm{CRD}}$ & $0.86(2)$ & $0.63(5)$ & $1.39(4)$ & $1.80(5)$ & & \\
\hline Manna & $0.84(2)$ & $0.60(3)$ & $1.40(2)$ & $1.80(5)$ & & \\
\hline \multirow[t]{3}{*}{ DP } & $0.81(1)$ & $0.581(5)$ & $1.39(3)$ & $1.901(5)$ & & \\
\hline & \multicolumn{6}{|c|}{ Spreading exponents $d=3$} \\
\hline & $\tau_{s}$ & $D$ & $\tau_{t}$ & $z$ & $\eta$ & $\delta$ \\
\hline CRD & $1.42(1)$ & $3.36(1)$ & $1.80(2)$ & $1.77(1)$ & $0.16(1)$ & $0.76(1)$ \\
\hline Manna & $1.41(1)$ & $3.36(1)$ & $1.78(2)$ & $1.76(2)$ & $0.16(5)$ & $0.78(2)$ \\
\hline DP & $1.359(1)$ & $3.507(1)$ & $1.730(1)$ & $1.901(5)$ & $0.114(4)$ & $0.730(4)$ \\
\hline
\end{tabular}

TABLE 1. Critical exponents for spreading and steady state experiments in $d=2$ and $d=3$. Models: CTTP: Conserved threshold transfer process; CRD: Conserved reaction-diffusion model; Manna: Abelian Manna sandpile; DP: Directed percolation (for the sake of comparison). 
jecture: all stochastic models with an infinite number of absorbing states in which the order parameter evolution is coupled to a static conserved field define a unique universality class [28]. This conjecture has been supported by extensive simulations of the following different models fulfilling all the above-listed requirements $[28,29]$ :

- (i) a conserved threshold transfer process [30,17];

- (ii) a conserved lattice gas with repulsion of nearest neighbor particles [28];

- (iii) a reaction-diffusion model (CRD) with two species A and B [29]

(see $[28,29]$ for model definitions and details). All the measured critical exponents both for steady state and for analysis of spreading [17] coincide within error bars and are also the same as those measured in extensive numerical simulations of the FES Manna sandpile [16] (up-to-date exponent values in two and three dimensions are listed in table I; for further simulation details as well as model and exponents definitions see $[16,28,29,17])$.

Furthermore, the slowly-driven versions of the previous models are self-organized $[28,29]$, and the corresponding avalanche exponents are in good agreement with those of stochastic sandpiles $[31,16]$. Finally, all the scaling laws among steadystate, spreading and avalanche critical exponents [32] are satisfied within numerical accuracy (except for some well identified anomalies $[16,28,29]$ ). This shows rather unambiguously that the critical point of a slowly-driven sandpile coincides with that of its FES version, supports strongly the previously enunciated conjecture, and identifies stochastic sandpiles as belonging in the universality class of systems with many absorbing states and order parameter coupled to a static conserved field.

Moreover, the conserved reaction-diffusion model studied in [29] has the notorious advantage of been susceptible to be mapped exactly using a a Fock-space representation and creation-annihilation operators [33] into an effective action, or equivalently into a set of Langevin equations [29]. Remarkably, up to naïvely irrelevant terms, this set of equations coincides with Eq.(1). The additional irrelevant terms appearing in the exact mapping, might also be of some importance in lower dimensions (this issue is currently under study).

\section{CONCLUSIONS AND OPEN PROBLEMS}

Different stochastic sandpile models show critical properties that are fully compatible with those of their corresponding FES versions. Using this equivalence stochastic sandpiles have been shown to belong in the universality class of systems exhibiting an absorbing-state phase transition with order parameter coupled to a static conserved density field. This result places sandpiles within the range of applicability of standard tools for the study of scale invariance in phase transitions, and constitutes in our opinion a step forward in the rationalizing SOC.

Finally let us enumerate some open questions and problems, on which we are presently working: 
- Direct numerical integration of the Langevin equations Eq.(1) using the method of [34]. This will provide an even more direct test for the validity of the Langevin equations.

- Connection of the universality class discussed in this paper with that of the pinning-depinning transitions in disordered systems [20]. Numerical simulations show that these two classes are indeed the same; we are working on the theoretical connections between them [35].

- Renormalization of the field theory for FES, Eq. (1).

- Clarification of some anomalies found in some time-dependent magnitudes in FES (like that affecting the exponent $\theta$ ).

- Extension of the numerical analysis to one-dimensional sandpiles.

ACKNOWLEDGMENTS We acknowledge M. Alava, A. Barrat, D. Dhar, P. Grassberger, K.B. Lauritsen, E.Marinari, L. Pietronero and A. Stella for useful discussions and comments. We acknowledge partial support from the European Network contract ERBFMRXCT980183; M.A.M acknowledges also support from the Spanish DGESIC project PB97-0842, and Junta de Andalucía project FQM165. R.D. acknowledges CNPq and CAPES. R.P.R. acknowledges support from grant CICYT PB97-0693.

\section{REFERENCES}

1. P. Bak, C. Tang and K. Wiesenfeld, Phys. Rev. Lett. 59, 381 (1987); Phys. Rev. A 38, 364 (1988).

2. S. S. Manna, J. Phys. A 24, L363 (1991).

3. Y.-C. Zhang, Phys. Rev. Lett. 63, 470 (1989).

4. D. Dhar, Phys. Rev. Lett.64, 1613 (1990); Physica A 263, 4 (1999). S. N. Majumdar and D. Dhar, Physica A 185, 129 (1992). For recent reviews see also D. Dhar, Physica A 264, 1 (1999); and cond-mat/9909009.

5. H. J. Jensen, Self organized criticality, (Cambridge Univ. Press, Cambridge, 1998).

6. G. Grinstein, in Scale Invariance, Interfaces and Nonequilibrium Dynamics, NATO Advanced Study Institute, Series B: Physics, vol. 344, A. McKane et al., Eds. (Plenum, New York, 1995). T. Hwa and M. Kardar, Phys. Rev. A 45, 7002 (1992). See also A. Barrat, A. Vespignani and S. Zapperi, Phys. Rev. Lett. 83, 1962 (1999).

7. M. De Menech, A. L. Stella and C. Tebaldi, Phys. Rev. E 58, R2677 (1998); C. Tebaldi, et al., Phys. Rev. Lett. 83, 3952 (1999). M. De Menech, A. L. Stella, Phys. Rev. E 62, R4528 (2000) See also; D. V. Ktitarev et al. Phys. Rev. E 61, 81 (2000). 
8. R. Dickman, M. A. Muñoz, A. Vespignani, and S. Zapperi, Braz. J. of Phys. 30, 27 (2000).

9. P. Bak and K. Sneppen, Phys. Rev. Lett. 71, 4083 (1993). K. Sneppen, Phys. Rev. Lett. 62, 3539 (1992). A. Hansen and S. Roux, J. Phys A 20, L873 (1987). P. Grassberger and Y.-C. Zhang, Physica A 224, 169 (1996). Z. Olami, I. Procaccia, and R. Zeitak, Phys. Rev. E 49, 1232 (1994). S. Maslov and Y-C. Zhang, Phys. Rev. Lett. 75, 1550 (1995). F. Bagnoli et al., Phys. Rev. E 55, 3970 (1997).

10. A. Vespignani and S. Zapperi, Phys. Rev. Lett. 78, 4793 (1997); Phys. Rev. E 57, 6345 (1998).

11. K. Chistensen, et al. Nature (London) 379, 49 (1996). L.A.N. Amaral and K. B. Lauritsen, Phys. Rev. E 54, 4512 (1996).

12. V. B. Priezzhev, J. Stat. Phys. 74, 955 (1994); E. V. Ivashkevich, J. Phys. A 27, 3643 (1994); E. V. Ivashkevich, et al., Physica A 209, 347 (1994). V. B. Priezzhev, cond-mat/9904054.

13. A. Díaz-Guilera, Europhys. Lett. 26, 177 (1994). L. Pietronero, A. Vespignani and S. Zapperi, Phys. Rev. Lett. 72, 1690 (1994). J. Hasty and K. Wiesenfeld, J. Stat. Phys. 86, 1179 (1997).

14. C. Tang and P. Bak, Phys. Rev. Lett. 60, 2347 (1988). See also, A. Montakhab and J. M. Carlson, Phys. Rev. E 58, 5608 (1998).

15. R. Dickman, A. Vespignani and S. Zapperi, Phys. Rev. E 57, 5095 (1998).

16. A. Vespignani, R. Dickman, M. A. Muñoz, and S. Zapperi, Phys. Rev. Lett. 81, 5676 (1998); Phys. Rev. E 62, 4564 (2000).

17. R. Dickman in Nonequilibrium Statistical Mechanics in One Dimension V. Privman, Ed. (Cambridge University Press, Cambridge 1996); G. Grinstein and M. A. Muñoz, in Fourth Granada Lectures in Computational Ed. P. Garrido and J. Marro, Lecture Notes in Physics, 493, 223 (Springer-Verlag, Berlin, 1997). J. Marro and R. Dickman, Nonequilibrium Phase Transitions in Lattice Models (Cambridge University Press, Cambridge, 1999). H. Hinrichsen, Nonequilibrium Critical Phenomena and Phase Transitions into Absorbing States, cond-mat/0001070.

18. Like any other statistical model, a fixed-energy sandpile exhibits critical singularities only in the infinite-size limit. In this limit the activity density is strictly zero for $\zeta<\zeta_{c}$, and positive for $\zeta>\zeta_{c}$, ensuring the stated inequality for $d \zeta / d t$ in the slowly-driven system.

19. Connections between absorbing state phase transitions and SOC models have been proposed in the past: M. Paczuski, S. Maslov, and P. Bak, Europhys. Lett. 27, 97 
(1994). S. Maslov and Y-C. Zhang, Physica A 223, 1 (1996). D. Sornett andI. Dornic, Phys. Rev. E 54, 3334 (1996). D. Sornette, et al., J. Phys. I (France) 5, 325 (1995). P. Grassberger, Phys. Lett. A200, 277 (1995). A. Vázquez and O. Sotolongo-Costa, J. Phys. A 32, 2633 (1999); Phys. Rev. E 61, 944 (2000).

20. O. Narayan and A. A. Middleton, Phys. Rev. B 49244 (1994). M. Paczuski and S. Boettcher, Phys. Rev. Lett. 77, 111 (1996). K. B. Lauritsen and M. Alava, condmat/9903346. M. Alava and K. B. Lauritsen, cond-mat/9902406.

21. D. Dhar and R. Ramaswamy, Phys. Rev. Lett. 63, 1659 (1989). B. Tadic and D. Dhar, Phys. Rev. Lett. 79, 1519 (1997).

22. S. Zapperi, K. B. Lauritsen, and H. E. Stanley, Phys. Rev. Lett. 75, 4071 (1995). E. V. Ivashkevich, Phys. Rev. Lett. 76, 3368 (1996).

23. J.L. Cardy and R.L. Sugar, J. Phys. A 13, L423 (1980); P. Grassberger, Z. Phys. B 47, 365 (1982); H.K. Janssen, Z. Phys. B 42, 151 (1981).

24. I. Jensen, Phys. Rev. Lett. 70, 1465 (1993); I. Jensen and R. Dickman, Phys. Rev. E 48, 1710 (1993).

25. M. A. Muñoz, G. Grinstein, R. Dickman and R. Livi, Phys. Rev. Lett. 76, 451, (1996). M. A. Muñoz, et al., J. Stat. Phys. 91, 541-569 (1998).

26. See: P. Grassberger et al., J. Phys. A 17, L105 (1984); N. Menyhard and G. Ódor, J. Phys. A 29, 7739 (1996); J. Cardy and U. C. Täuber, Phys. Rev. Lett. 77, 4780 (1996); H. Hinrichsen, Phys. Rev. E 55, 219 (1997); W. Hwang, S. Kwon, H. Park, and H. Park, Phys. Rev. E 57, 6438 (1998); and references therein.

27. R. Pastor-Satorras and A. Vespignani, cond-mat/0010223. In press (Eur. Phys. J. B.)

28. M. Rossi, R. Pastor-Satorras and A. Vespignani, Phys. Rev. Lett. 85, 1803 (2000).

29. R. Pastor-Satorras and A. Vespignani, Phys. Rev. E62 , R5875 (2000). The model studied in this reference is a limiting case of a more general class studied in F. van Wijland, K. Oerding, and H. J. Hilhorst, Physica A251 , 179 (1998).

30. This is a conserved counterpart of the model introduced in: J.F.F Mendes et al. J. Phys. A 27, 3019 (1994).

31. P. Grassberger and S. S. Manna, J. Phys. (France) 51, 1077 (1990). S. S. Manna, J. Stat. Phys. 59, 509 (1990). S. Lübeck and K.D. Usadel, Phys. Rev. E 55, 4095 (1997); ibid. 56, 5138 (1997).

32. See, for instance, M. A. Muñoz, R. Dickman, A. Vespignani, and S. Zapperi, Phys. Rev. E 59, 6175 (1999); and references therein. 
33. M. Doi, J. Phys. A9, 1465 (1976). L. Peliti, J. Physique 46, 1469 (1985). B. P. Lee and J. Cardy, J. Stat. Phys. A 80, 971 (1995).

34. R. Dickman, Phys. Rev. E 50, 4404 (1994). C. López and M. A. Muñoz, Phys. Rev. E 56, 4864 (1997).

35. M. Alava and M. A. Muñoz, In preparation. 